\title{
Recombinant tissue plasminogen activator treatment for intracardiac vegetation in a very low birth weight infant
}

\author{
Abdurrahman A. Ozdemir, M.D. ${ }^{a}$
}

\begin{abstract}
Developing treatment options have resulted in prolonged admission and increased bacterial and fungal nosocomial infections as well as improved survival in neonatal intensive care unit. Invasive fungal infections in newborns are associated with significant morbidity and mortality and can cause endorgan dissemination such as endocarditis, endophthalmitis, septic arthritis, obstructive nephropathy and meningitis. Endocarditis requires aggressive systemic antifungal therapy and sometimes surgical intervention in neonates.

We reporta low birth weight prematüre infant with intracardiac vegetation that is rare and a life-threatening complication of invasive fungal infections. He was treated with caspofungin and recombinant tissue plasminogen activator in stead of surgical intervention.

Key words: mycotic, endocarditis, recombinant tissue activator, caspofungin.
\end{abstract}

http:/ / dx.doi.org/10.5546/ aap.2017.eng.e307

To cite: Ozdemir AA. Recombinant tissue plasminogen activato treatment for intracardiac vegetation in a very low birth weight infant. Arch Argent Pediatr 2017;115(5):e307-e310.

\section{INTRODUCTION}

Developing treatment options have resulted in prolonged admission and increased nosocomial fungal infections as well as improved survival in neonatal intensive care units (NICUs). Candida spp. is the third most common cause of late-onset infections in very low birth weight (VLBW) and extremely low birth weight (ELBW) infants with an incidence ranging from 2.6 to $10 \%$ and from 5.5 to $20 \%$, respectively. The mortality rate of invasive fungal infections has been reported as high as $20-60 \%$ in preterm infants. ${ }^{1-3}$

a. Department of Pediatrics, Division of Neonatology, Istanbul Medicine Hospital, Istanbul, Turkey.

E-mail address:

Abdurrahman A. Ozdemir, M.D.: avarozdemir@gmail.com

Funding: None.

Conflict of interest: None.

Received: 1-14-2017

Accepted: 5-6-2017
Fungal endocarditis is a life-threatening complication of invasive Candida spp. infections in infants. It requires long-term systemic antifungal therapy and sometimes surgical interventions. ${ }^{4,5}$ Herein, we report a VLBW infant with intracardiac fungal vegetation that was succesfully treated with caspofungin and recombinant tissue plasminogen activator (rTPA).

\section{CASE REPORT}

A premature male infant was born at $28^{\text {th }}$ week of gestation because of fetal distress, with a birth weight of $1030 \mathrm{~g}$ from a 30 years old mother's second pregnancy. The patient was transferred to NICU and early surfactant therapy was given after intubation for respiratory distress syndrome. After umbilical catheter was inserted, ampicillin and gentamicin were administered because of sepsis could not be excluded. Fluconazole was started for antifungal prophylaxis due to increased risk factors. On the $3^{\text {rd }}$ day, he presented a pulmonary hemorrhage; therefore enteral feeding was stopped, surfactant and fresh frozen plasma (FFP) were given. Minimal enteral feeding was started on the $8^{\text {th }}$ day. The day after, patient's condition got worse with abdominal distention. Direct X-ray and ultrasonography showed dilated bowel loops, especially in the ileocolic region. At that time, laboratory data revealed leukocytes $17460 / \mathrm{mm}^{3}$, platelets $107000 / \mathrm{mm}^{3}$, normal CRP $(0.3 \mathrm{mg} / \mathrm{dL})$ level and hyperglycemia $(159 \mathrm{mg} / \mathrm{dL})$. He was diagnosed as necrotizing enterocolitis (NEC); antibiotics were changed to teikoplanin, cefoperazone-sulbactam and metronidazole; enteral feeding was stopped again. On the $14^{\text {th }}$ day, the patient was extubated and nasal CPAP was started. After 3 days, apnea and metabolic acidosis emerged and he was reintubated. A sepsis work-up was performed and $C$. parapsilosis was identified in his blood culture. Thus, umbilical catheter was removed and conventional amphotericin B was started. Repeated cranial and abdominal ultrasounds, $\mathrm{ECHO}$, eye examination were found normal. The following week, Candida parapsilosis continued to grow in his blood cultures and ECHO showed a mass of $7.7 \mathrm{~mm}$ in length and $7.3 \mathrm{~mm}$ in 
width (fungal ball) at right atrium (Figure 1). Addionally, his lower extremities began to swell due to venous flow obstruction. Thus, caspofungin acetate $(2 \mathrm{mg} / \mathrm{kg} /$ day $)$ was added to conventional amphotericin B for combined therapy. After 8 days, fungal growth was not detected in his cultures, so amphotericin B and caspofungin were administered for a total of 6 weeks. Follow-up blood cultures remained negative. However, the size of the vegetation did not changed as well as the swelling of lower extremities continued in following days. Hence, it was decided to use rTPA for thrombolysis. After the treatment protocol was approved by the ethics committee and informed consent was obtained from his parents, rTPA therapy was infused at $0.3 \mathrm{mg} / \mathrm{kg} / \mathrm{h}$ for $6 \mathrm{~h}$ after fresh frozen plasma (FFP) was given to patient. Before treatment, cranial USG was found to be normal and basal coagulation parameters (PT, aPTT, INR and fibrinogen) were within normal range. rTPA was repeated for a total of 3 days under close monitoring. Serial ECHOs revealed that the size of the vegetation regressed to $2.1 \mathrm{~mm} \times 2.5 \mathrm{~mm}$ and no complications were observed (Figure 2). After rTPA treatment, venous obstruction resolved and the swelling in his extremities disappeared. He was discharged on the $84^{\text {th }}$ day of admission and he is now being followed by the Department of Pediatric Cardiology.

\section{DISCUSSION}

The most common cause of fungal infections in newborns is Candida spp.; Candida albicans (C. albicans) and Candida parapsilosis (C. parapsilosis) are account for the most of invasive Candida infections in NICU. ${ }^{1,6}$

FIGURE 1. Echocardiography showing the vegetation in the right atrium before rTPA treatment

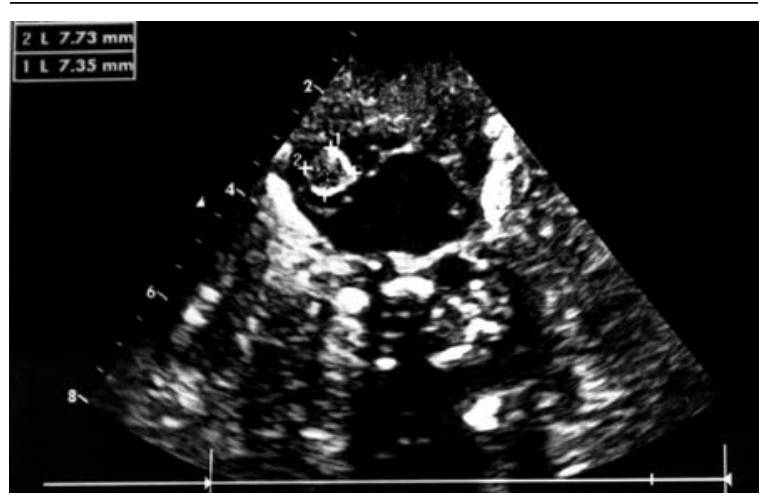

Many risk factors, such as prematurity, low birth weight, prolonged intubation, delay in enteral feeding, broad spectrum antibiotics, steroid and $\mathrm{H} 2$ blocker usage, surgical interventions, NEC and prolonged usage of central catheter increase the frequency of fungal infections. ${ }^{7}$ The majority of the risk factors were present in our case and C. parapsilosis was isolated in his blood culture.

Candida spp.can cause end-organ dissemination such as endocarditis, endophthalmitis, obstructive nephropathy and meningitis. The frequency of cardiac involvement in invasive fungal infections has been reported between 5 to $15 \%$ and it has very high mortality. Therefore, early diagnosis and aggressive management are very important for succesful treatment. Fungal endocarditis may be associated with intracardiac vegetations in the right heart in newborns. Especially, endothelial damage and central catheters contributes to the pathogenesis of endocarditis and formation of thrombus. When candidemia is determined, central venous catheter should be removed and ECHO must be performed as well as fundoscopy, CSF, urine analysis. ${ }^{14,5,8}$ After Candida spp. was detected, we started antifungal treatment and removed the umbilical catheter. All of systems were evaluated for end-organ dissemination and $\mathrm{ECHO}$ showed fungal vegetation in right atrium.

The treatment of invasive candidiasis requires prolonged use of antifungal agents such as fluconazole, amphotericin B formulations (conventional and liposomal), echinocandins (micafungin and caspofungin) for at least 4-6 weeks or until 2 weeks after 2 negative blood culture. Fluconazole is a

FIGURE 2. Echocardiography showing the shrinkage of the vegetation after 1 week of rTPA treatment

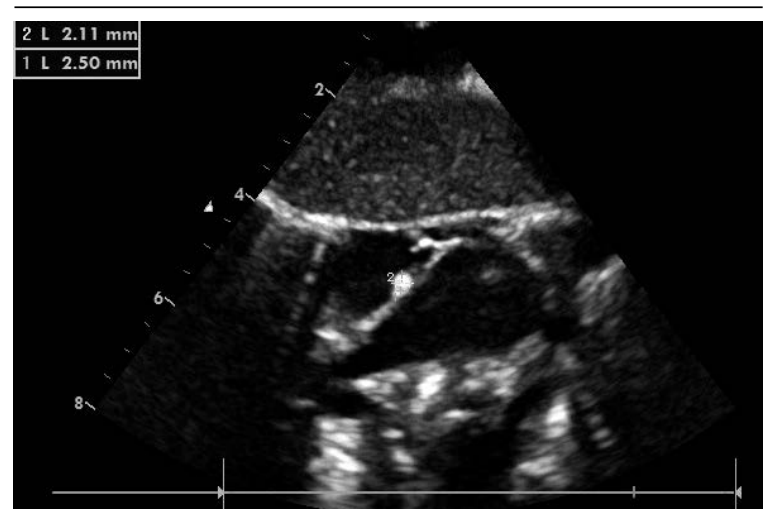


fungistatic agent with narrow spectrum, therefore amphotericin B formulations and echinocandins are recommended in the initial treatment of Candida spp. endocarditis in neonates. However, the appropriate dosage for caspofungin has not been clearly defined in neonates. The recommended infant dosage of caspofungin $\left(25 \mathrm{mg} / \mathrm{m}^{2} /\right.$ day or $1-2 \mathrm{mg} /$ $\mathrm{kg} /$ day) is based on a small number of case series..$^{9-11}$ Antifungal agents may be used in combination inthe treatment of endocarditis. In a review study, Pana et al. reported that combined antifungal treatment was associated with lower mortality than monotherapy. ${ }^{5}$ In our patient, conventional amphotericin B was administered for initial therapy. In following days, Candida spp. continued to grow in his blood cultures. Thus, caspofungin was added for combined therapy. After 8 days, Candida spp. was not isolated in cultures; caspofungin and amphotericin $\mathrm{B}$ were administered for a total of 6 weeks.

In our patient, there was no change in the size of the intracardiac vegetation and blood flow obstruction, although the cultures were negative with caspofungin treatment. In such cases, the treatment of fungal endocarditis requires use of antifungal agents with surgical removal of thrombus. However, the decision of surgical intervention in preterm infants is difficult because of high mortality risk due to clinic instability and very low birth weight. ${ }^{1,4,5}$ Therefore, we decided to use thrombolytic therapy instead of surgical intervention. Urokinase, streptokinase and rTPA are used for thrombolytic therapy in children and adults. However, urokinase and streptokinase are not generally preferred in the neonatal period due to decreased fibrin specicity and high antigenity. ${ }^{12}$ rTPA is a recombinant protein that catalyzes the conversion of plasminogen to plasmin. It has some advantages such as low antigenicity, direct activation of plasminogen, fibrinolysis without causing proteolysis and a short half-life compared with streptokinase and urokinase. ${ }^{13}$ Consequently, we decided to use rTPA for tromboliytic therapy, although there are very limited data about rTPA treatment in preterms with intracardiac fungal thrombus.

rTPA was generally used $0.1-0.6 \mathrm{mg} / \mathrm{kg} / \mathrm{h}$ for 6-12 $\mathrm{h}$ and was repeated for 3 days, although the dose and duration varies in different studies. , $^{813-15}$ Before thrombolytic treatment, the patients must be evaluated for contraindications that include seizures within 48 hours, active bleeding, coagulation deficiency, invasive procedures within 3 days, thrombocytopenia, low fibrinogen levels, hypertension and severe sepsis. ${ }^{15}$ In our case, there were no contraindications for trombolytic teatment, so we started rTPA treatment after FFP was administered for providing sufficient plasminogen. At the end of 3 days, ECHO revealed that the size of vegetation significantly decreased and rTPA treatment was discontinued. Thus, intracardiac vegetation was succesfully treated with average dosage and duration of rTPA and there was no complications during and after rTPA treatment in our case.

In conclusion, we suggest that caspofungin could be an alternative treatment option in preterm infants with Candida spp. endocarditis refractory to other antifungals. Additionally, fungal vegetation was succesfully treated with rTPA. This case showed that rTPA could be used with close monitoring in the treatment of intracardiac thrombus and it might be an important alternative to surgical intervention in the high-risk infants. However, larger and prospective studies are required to determine the safe dosage and duration of the rTPA treatment in preterm infants.

\section{REFERENCES}

1. Kaufman DA, Fairchild KD. Clinical microbiology of bacterial and fungal sepsis in very-low-birth-weight infants. Clin Microbiol Rev 2004;17(3):638-80.

2. Zaoutis TE, Heydon K, Localio R, etal.Outcomes attributable to neonatal candidiasis. Clin Infect Dis 2007;44(9):1187-93.

3. Montagna MT, Lovero G, De Giglio O, et al. Invasive fungal infections in neonatal intensive care units of southhern Italy: a multicentre regional active surveillance (AURORA project). J Prev Med Hyg 2010;51(3):125-30.

4. NoyolaDE,FernandezM,MoylettEH, etal.Ophthalmologic, visceral, and cardiac involvement in neonates with candidemia. Clin Infect Dis 2001;32(7):1018-23.

5. Pana ZD, Dotis J, Iosifidis E, et al. Fungal endocarditis in neonates: A review of seventy-one cases(1971-2013). Pediatr Infect Dis J 2015;34(8):803-8.

6. López Sastre JB, Coto Cotallo GD, Fernández Colomer B. Neonatal invasive candidiasis: a prospective multicenter study of 118 cases. Am J Perinatol 2003;20(3):153-63.

7. Swanson JR, Gurka MJ, Kaufman DA. Risk factors for invasive fungal infection in prematüre infants: Enhancing a targeted prevention approach. J Pediatric Infect Dis Soc 2014;3(1):49-56.

8. Babayigit A, Cebeci B, Buyukkale G, et al. Treatment of neonatal fungal infective endocarditis with recombinant tissue plasminogen activator in a low birth weight infant case report and review of the literature. Mycoses 2015;58(10):578-81.

9. Santolaya ME, Alvarado Matute T, de Queiroz Telles F, et al. Recommendations for the management of candidemia in neonates in Latin America. Latin America Invasive Mycosos Network. Rev Iberoam Micol 2013;30(3):158-70.

10. Hope WW, Castagnola E, Groll AH, et al. ESCMID guideline for the diagnosis and management of Candida diseases 2012: prevention and management of invasive infections in 
neonates and children caused by Candida spp. Clin Microbiol Infect 2012;18 Sippl 7: 38-52.

11. Lestner JM, Smith PB, Cohen-Wolkowiez M, et al. Antifungal agents and therapy for infants and children with invasive fungal infections: a pharmacological perspective. Br J Clin Pharmacol 2013;75(6):1381-95.

12. Veldman A, Nold MF, Michel-Behnke I. Thrombosis in the critically ill neonate: incidence, diagnosis, and management. Vasc Health Risk Manag 2008;4(6):1337-48.

13. Hartmann J, Hussein A, Trowitzsch E, et al. Treatment of neonatal thrombus formation with recombinant tissue plasminogen activator: six years experience and review of the literature. Arch Dis Child Fetal Neonatal Ed 2001; 85(1):F18-22.

14. Ferrari F, Vagnarelli F, Gargano G, et al. Early intracardiac thrombosis in preterm infants and thrombolysis with recombinant tissue type plasminogen activator. Arch Dis Child Fetal Neonatal Ed 2001;85(1):F66-9.

15. Aydin B, Beken S, Dilli D, et al. Fibrinolytic therapy of thrombosis in 27 newborns followed-up in neonatal intensive care unit. Pediatr Hematol Oncol 2013;30(8):705-16. 